\title{
Modelling of the in-flight synthesis of TaC nanoparticles from liquid precursor in thermal plasma jet
}

\author{
A Vorobev ${ }^{1}$, O Zikanov and P Mohanty \\ Department of Mechanical Engineering, University of Michigan-Dearborn, 48128-1491, \\ Dearborn, MI, USA \\ E-mail: pmohanty@umd.umich.edu
}

Received 1 October 2007, in final form 15 January 2008

Published 12 March 2008

Online at stacks.iop.org/JPhysD/41/085302

\begin{abstract}
A simple and efficient numerical model describing the processes of nucleation, growth and transport of multi-component nanoparticles is developed. The approach is conceptually similar to the classical method of moments but can be applied to co-condensation of several substances. The processes of homogeneous nucleation, heterogeneous growth, and coagulations due to Brownian collisions are considered in combination with the convective and diffusive transport of particles and reacting gases within multi-dimensional geometries. The model is applied to the analysis of multi-component co-condensation of $\mathrm{TaC}$ nanoparticles within a dc plasma reactor.
\end{abstract}

\section{Introduction}

Thermal plasma spraying is a popular method for production of thin coatings made of different materials and having diverse applications. The precursor materials are predominantly provided in powder form with a desired composition. One example is the refractory coating, for which the method works well due to extremely high temperatures generated by a plasma discharge. In this paper we focus on the production of tantalum carbide (TaC) coating from liquid, rather than powder, precursors. $\mathrm{TaC}$ with the melting point of $3880{ }^{\circ} \mathrm{C}$ is one of the hardest substances used for cutting and boring machine tools, as well as for high-temperature wear-resistant applications (see Fetherston 2004, Ishigaki et al 2005).

The properties of coating are strongly affected by the parameters of the deposition process. In particular, it is highly desirable that the particles of approximately the same (and better small) size and uniform chemical composition are deposited (see, e.g. Trignan-Piot et al 1996). The recent trend is to obtain the coating from nanosize particles. Although this concept is promising, its realization introduces new challenges into the existing technology.

The use of liquid precursors was recently identified as a possible way of production of nanophase coatings

${ }^{1}$ Present address: University of Southampton, School of Engineering Sciences, Southampton, SO17 1BJ, UK.
(Killinger et al 2006). The liquid precursor is usually a solution of the desired material in water or an organic solvent. The droplets are injected into the hot plasma zone, where the precursor is evaporated and chemically decomposed into a mixture of elemental gases. The hot gas leaving the plasma gun is cooled down by the surrounding air. Nanoparticles are nucleated in the quenching zone and transported by the jet to the target substrate.

The feasibility of producing coating with nanosize particles using liquid precursors was demonstrated in several experimental studies (e.g. Hafiz et al 2004, Ishigaki et al 2005, Killinger et al 2006). Nevertheless, some technical difficulties still exist, such as, the problem of efficient deposition of almost inertialess particles onto the substrate. Today, this method is more successful in production of nanoparticles (e.g. Ishigaki et al 2005, Killinger et al 2006) than in formation of coatings.

In this paper, we consider solely the production of nanoparticles in the environment of a dc plasma jet. The problem is approached using mathematical and computational modelling. A complete model of the process should include at least three components: (i) a model of the gas flow; (ii) a model of the injection of liquid precursor droplets and their subsequent evaporation and (iii) a model of formation of new particles, their transport by the flow, and their growth via condensation and coagulation. The main focus of this paper is on the last of these steps. We develop a numerical model, which 
accurately captures the physics of the process and, at the same time, is feasible numerically.

There have been numerous works devoted to modelling of the synthesis of particles in plasma reactors (see, e.g. Desilets et al 1997, Mendoza-Gonzalez et al 2007, McFeaters et al 1994, Murphy 2004). Some of the models are quite comprehensive and include 2D modelling of coupled hydrodynamic and electromagnetic equations, species transport, chemical reactions, nucleation, condensation, coagulation and some other effects. These studies, however, are focused on the nucleation and growth of particles consisting of one chemical component. The method of moments is often applied to describe the particle nucleation and evolution.

The novelty of our work is the extension of the analysis to the situation when the particles are formed by co-condensation of two chemically different gases (Ta and $\mathrm{C}$ in our case). One of the few works, where the modelling of a similar process was conducted, were the papers of Shigeta and Watanabe (2007a, 2007b), where the formation of boron- and silicon-based particles in an RF plasma reactor was examined. The authors proposed a co-condensation model, in which particles are formed by homogeneous nucleation of one gas and subsequent heterogeneous condensation of two gases. The model was applied to individual particles following onedimensional pathlines. The innovative approach to the main subject, the particle formation, was combined with several rather strong simplifying assumptions. In particular, the effects of multi-dimensional turbulent transport and diffusion, particle collisions, and the compressibility of the gas flow were neglected.

In this paper, we combine a more realistic hydrodynamic approach, 2D analysis of transport and mixture of particles and gas species, and coagulation processes with consideration of co-condensation of two-component particles. The model describing the particle nucleation and evolution is based on the idea of the method of moments commonly used in the aerosol science. The key component of the method is consideration of several first moments of particle density function. The concept is expanded to the case of the formation of two-component particles. The model developed includes the processes of homogeneous nucleation, heterogeneous condensation and Brownian coagulation. The model gives predictions for the number of particles formed, their mean size and chemical composition.

\section{System under consideration and typical parameters}

The model developed in this paper describes synthesis of $\mathrm{TaC}$ particles in a jet of a dc plasma gun. The analysis includes certain features specific to this particular system, such as extremely high temperature within the nozzle and high flow velocity. On the other hand, the general modelling approach can be adapted to other systems of nanoparticle production based on the same physical principle, namely, on quenching to physical conditions that cause homogeneous nucleation and subsequent heterogeneous condensation.
The parameters of the dc plasma gun installed in our laboratory are used for the simulations. Pure argon is injected through the inlet with the mass flux of $1.68 \times 10^{-3} \mathrm{~kg} \mathrm{~s}^{-1}$. Within the plasma gun, the argon is heated by a dc plasma arc. The operating voltage of the gun is set at $38-40 \mathrm{~V}$, and the operating current is $600 \mathrm{~A}$. The heated and partially ionized gas is issued into the open atmosphere at room temperature. As a result, the hot argon gas is mixed and cooled by surrounding air.

The formation of $\mathrm{TaC}$ nanoparticles is initiated by injection of the liquid precursor, tantalum ethoxide, $\mathrm{Ta}\left(\mathrm{OC}_{2} \mathrm{H}_{5}\right)_{5}$ (see, e.g. Ishigaki et al 2005) into the reactor. The temperature of the jet is sufficiently high to evaporate the precursor and dissociate the atoms of its molecules, which leads to formation of monoatomic gases of Ta and C. A target substrate, where the coating should be formed, is typically set up at a distance of about $10 \mathrm{~cm}$ from the nozzle.

As discussed in detail in the following sections, some simplifying modifications need to be made in the theoretical model in comparison with the actual gun. This, in particular, concerns the way in which the discharge arc and other processes within the nozzle are simulated. Furthermore, in accordance with the focus of our study on the formation of particles, the target substrate is removed in the model.

\section{Preliminary steps}

\subsection{Modelling of the gas flow}

There have been many works that attempted to describe the gas flow generated by a dc plasma gun. The main difficulty was to find a correct description of the plasma torch structure and behaviour. Three different operation modes of the torch are usually distinguished (see Trelles et al 2006): steady mode, takeover mode and restrike mode, each corresponding to a particular pattern of arc movement. The numerical models found in the literature can relatively accurately predict the structure of the torch and the flow generated for the steady mode only. The most desirable and used regime of operation of the plasma reactor is, however, the takeover mode. The arc in this regime is moving periodically or quasi-periodically over the anode's surface, thus securing a more homogeneous distribution of the heat load than in the case of the steady mode. On the other hand, the arc movement is more predictable and, consequently, allows more predictable results to be obtained than in the regime of the restrike mode.

In a recent study, Trelles et al (2006) attempted to obtain the structure of the jet induced by the torch in its takeover mode of operation. The results obtained gave a correct qualitative description of the torch and flow pattern. The quantitative agreement with the experimental data was, however, not achieved. This does not seem surprising if one considers the difficulties involved in the accurate computational modelling of a turbulent magnetohydrodynamic plasma flow with very large gradients of temperature and velocity and the significant role played by the real plasma physics. The task can be accomplished in the future and requires a separate extensive investigation.

Since the focus of our investigation is on the formation of nanoparticles rather than on the arc and jet behaviour we 
chose to obtain the needed properties of the jet flow by using a simple hydrodynamic model correlated with experimental data. A similar approach was followed in earlier studies of nanoparticle formation, e.g. McFeaters et al (1994) and Murphy (2004). In other studies, such as e.g. Basu and Cetegen (2007), the velocity and temperature fields were taken directly from the measurements. Although clearly an approximation of the actual nozzle and jet flow, our model is believed to be sufficient for the purposes of this work.

The system of the Navier-Stokes equations for a compressible, viscous and heat-conducting mixture of ideal gases (argon and air) is solved numerically using the CFD software Fluent. The ideal gas law is used as the state equation. The thermodynamic and transport coefficient of a mixture of argon and air are determined via the ideal-gas-mixing formulae (these mixing formulae are based on the kinetic theory and can be found in Fluent 6.3 User's Guide).

Drastic (but inevitable) simplifications are made in the modelling of the arc discharge. We do not try to reproduce the electromagnetic effects and the arc dynamics. Ionization of the gas is not considered. Instead, the effect of the discharge on the gas flow is imitated by input from a heat source located within the plasma gun. To justify the approach we mention that the nanoparticle formation occurs entirely outside the nozzle. One can assume that the parameters of the jet flow in that area are primarily determined by the rate of energy generation within the nozzle. The details of the mechanism of the energy generation can be neglected in the first approximation.

The power of the heat source is chosen to be $16.2 \mathrm{~kW}$, which turned out to be the maximum power at which a convergent steady solution for the gas flow could be obtained. The total heat-conduction losses through anode and cathode are $4.1 \mathrm{~kW}$. The power used for the heat source is small compared with the typical powers at which the dc plasma reactors operate (22.8-24 kW for the set-up in our laboratory). It can be assumed that the difference accounts for the losses not taken into account directly in the model, such as radiation losses. In general, it can be noted that the thermal efficiency of a plasma gun is significantly lower than $100 \%$. For instance, in the experimental study of Vardelle et al (1983), it was estimated as $72 \%$ or $63 \%$ depending on the regime of operation.

The energy generation rate is distributed within the nozzle interior with the density:

$$
E=E_{0} \exp \left(-2\left(\frac{s}{s_{0}}\right)^{5}\right)
$$

where the constant $E_{0}=1.35 \times 10^{10} \mathrm{~J} \mathrm{~m}^{-3}$, and $s$ is the distance from the tip of the cathode $\left(z=z_{0}, r=0\right.$ ) (see figure $1(a)), z_{0}=2.5 \mathrm{~cm}$, and $s_{0}=z_{0} / 3$. Similar distribution is assumed by Trelles et al (2006) for the density of current near the cathode's tip.

The outcome of the computations is a set of ensembleaveraged (mean) steady-state fields of temperature, velocity, concentrations of argon and air, pressure and density. The effects of turbulent fluctuations on the flow and mixing is taken into account via the turbulent eddy viscosity and diffusivity coefficients modelled by the realizable $k-\epsilon$ turbulence model (Shih et al 1995). The temperature dependence of thermodynamic and transport coefficients of argon is accounted for by the use of the empirical formulae available in the papers by Liu et al (2000) and Chen et al (1996).

The entire computational domain is shown in figure $1(a)$. The geometry and dimensions of the problem reproduce the parameters of the dc plasma gun used in our laboratory. The problem is solved under the assumption of axial symmetry. In accordance with our focus on synthesis of nanoparticles rather than on the actual coating process, the target wall is removed. Free flow of gases is assumed at the outlet boundary, which is taken sufficiently far from the nozzle $(z=20.32 \mathrm{~cm})$ so that the boundary does not affect the flow in the area, where the key processes of particle formation occur.

Gas pressure is maintained at $1.5 p_{\mathrm{atm}}$ at the inlet into the nozzle $(z=0)$ and at $p_{\text {atm }}$ at the open boundaries $r=5.08 \mathrm{~cm}$ and $z=20.32 \mathrm{~cm}$, where $p_{\text {atm }}$ is the atmospheric pressure. Throughout the entire domain outside the nozzle, the gas pressure is found to be close to atmospheric. For the gas velocity, the standard wall-functions (Launder and Spalding 1974) are used as the velocity boundary conditions on the solid walls of the nozzle. This provides the effective description of the turbulent boundary layers. At the inlet, the mass flux of $1.68 \times 10^{-3} \mathrm{~kg} \mathrm{~s}^{-1}$ of pure argon at temperature $300 \mathrm{~K}$ is imposed (the 'mass-flow-inlet' boundary condition in terms of Fluent 6.3). At the open boundaries the flow is assumed to be settled, and the reverse flux of pure air with temperature $300 \mathrm{~K}$ is permitted (the 'pressure-outlet' boundary condition of Fluent 6.3).

One source of energy losses, namely the heat loss through the reactor walls, is directly simulated in our model. At the surface of the anode, the convection boundary conditions are imposed. Heat transfer coefficient is taken to be $10^{5} \mathrm{~W} \mathrm{~m}^{-2} \mathrm{~K}^{-1}$. The cathode boundary temperature is constant in time and varies from $300 \mathrm{~K}$ at the inlet to $1000 \mathrm{~K}$ at the cathode tip, according to the following profile:

$$
T=300+700 \exp \left(-2\left(\frac{s}{s_{0}}\right)^{5}\right) .
$$

The thermal boundary conditions described above were partly adopted from the paper of Trelles et al (2006). It should be understood that such a set of boundary conditions is only an attempt of a reasonable approximation needed in the absence of reliable experimental or computational data. We investigated the possible effect of inaccuracy in the thermal boundary conditions on the temperature and velocity of the jet by varying the parameters, such as the maximum temperature of the cathode surface, heat transfer coefficient for the anode wall, value of the inlet pressure, etc. No significant effect was found for plausible (not very large) variations.

The results of numerical calculations for the case chosen for further analysis are shown in figure 1. Despite the number of very strong approximations listed above, the typical values of the calculated physical quantities and their distributions in space are in reasonable agreement with the experimental measurements (see, e.g. Vardelle et al 1983). Most importantly for the following analysis, one can see the rapid decrease of 

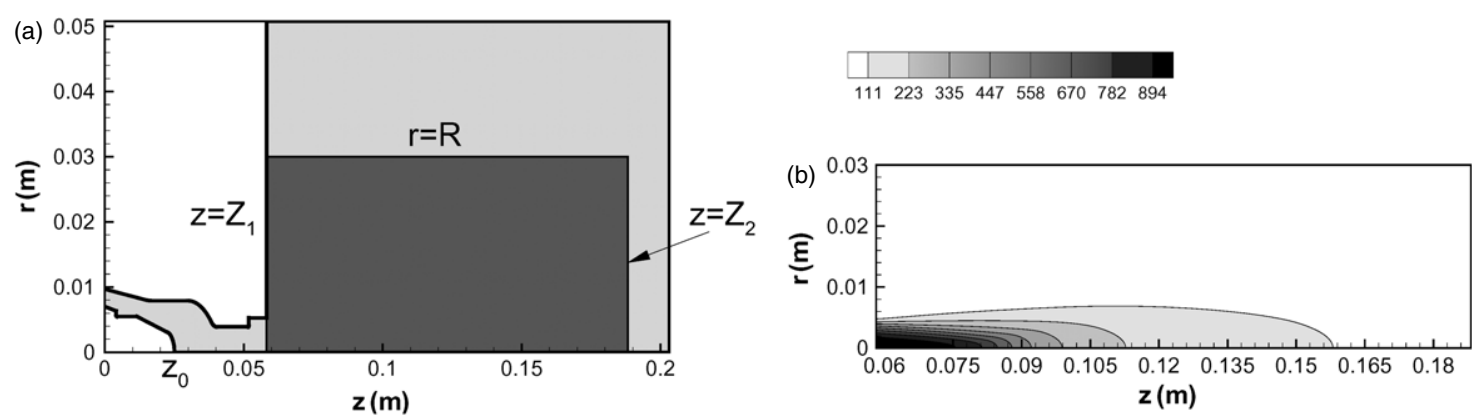

(c) \begin{tabular}{lllllllll}
\hline & & & & & & \\
$2.0 \mathrm{E}+03$ & $3.6 \mathrm{E}+03$ & $5.3 \mathrm{E}+03$ & $6.9 \mathrm{E}+03$ & $8.6 \mathrm{E}+03$ & $1.0 \mathrm{E}+04$ & $1.2 \mathrm{E}+04$ & $1.4 \mathrm{E}+04$
\end{tabular}

(d)
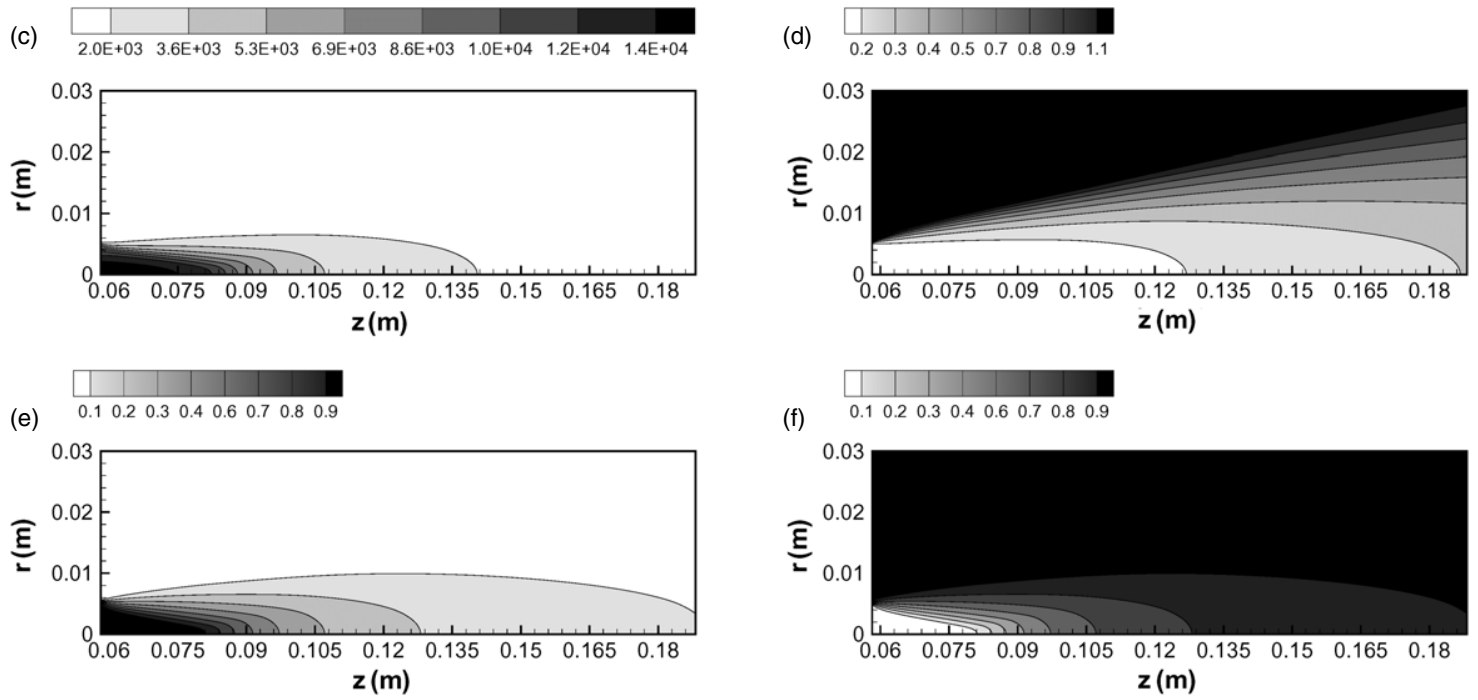

Figure 1. Results of the simulations of the gas flow. (a) Full computational domain (light-grey) and the domain where the evolution of particles is studied (dark-grey). Distributions of $(b)$ axial velocity $\left(\mathrm{m} \mathrm{s}^{-1}\right),(c)$ temperature $(\mathrm{K}),(d) \mathrm{gas}$ density $\left(\mathrm{kg} \mathrm{m}^{-3}\right)$ and mass fractions of $(e)$ argon and $(f)$ air.

temperature of the gas (figure $1(c)$ ) that occurs due to the turbulent heat transfer and intensive mixing of argon with surrounding air illustrated in figures $1(e)$ and $(f)$.

The last comment concerns yet another simplification used in our model of the gas flow. We assume that the mass ratio between the flow rates of the precursor and argon gas is much smaller than one. As a result, we can neglect the effect of the added material, in particular, the effects of liquid precursor droplets and the heat of their evaporation, on the gas flow. The assumption limits the maximum loading by precursor and, eventually, the maximum concentrations of free atoms of $\mathrm{Ta}$ and $\mathrm{C}$ available for formation of nanoparticles, at which our model produces accurate results.

\subsection{Injection of precursor}

There are two principal ways to inject the precursor into the plasma reactor: axisymmetric injection, usually carried out at the inlet opening, and lateral injection, when the precursor is added laterally close to the gun outlet. The lateral injection can be more easily realized although additional care is needed to arrange the penetration of particles into the core of the jet without adding strong disturbances to the main flow.

In principle, one can apply the methods of CFD to numerically model the evolution of the injected precursor droplets, which includes transport by the flow, heating and evaporation. In the particular case considered in this paper, accurate simulations are impossible because the data on key thermodynamic properties of tantalum ethoxide, such as heat conductivity, heat capacity, heat of vaporization, saturation vapour pressure and surface tension, are unavailable in the literature. The stages of chemical decomposition are also unknown. We circumvent these obstacles by assuming that owing to high temperatures in the interior of the plasma gun and near the nozzle, the precursor is entirely evaporated before the conditions suitable for formation of nanoparticles are achieved.

The decomposition of precursor results in a mixture of several elemental gases in the region adjoining the exit from the nozzle. One should expect, especially due to the presence of hydrogen, that hundreds of chemical reactions occur with formation of hundreds of new chemical species. We are solely interested in the concentration of tantalum and carbon species. For this reason, the simplified approach is taken, according to which certain concentrations of Ta and $\mathrm{C}$ vapours are imposed as inlet conditions at the inlet boundary of the computational sub-domain used for the investigation of the particle evolution (the dark-grey area in figure 1(a)). Owing to the simplifying assumptions, the values of these concentrations cannot be calculated and should be prescribed as input parameters. Moreover, these values can only be roughly estimated from the value of mass influx of the precursor, since the chemical reactions, such as, e.g. the synthesis of $\mathrm{CO}$, which behaves essentially as an inert gas (McFeaters et al 1994), can eliminate 
significant amount of carbon from the process of particle formation. Furthermore, certain fraction of free tantalum or carbon can be moved out of the domain of investigation by turbulent fluctuations.

To conclude this subsection, let us estimate the allowed concentrations of the $\mathrm{Ta}$ and $\mathrm{C}$ gases in the region close to the nozzle outlet. Our calculations for the main gas flow show that the value of pressure is close to atmospheric throughout the whole computational domain, where formation of particles is considered. The molecular concentration of the carrier gas mixture can be estimated as $p /(k T) \approx 10^{24} \mathrm{~m}^{-3}$ (here $k$ is the Boltzmann constant). This means the maximum concentrations of Ta and $\mathrm{C}$, still satisfying the assumption of low precursor/gas ratio, can be estimated as $10^{22} \mathrm{~m}^{-3}$.

It is easy to estimate the mass flux of the precursor from the inlet concentrations of Ta and $\mathrm{C}$. If we neglect the diffusion transport at the inlet boundary, and assume that $\mathrm{Ta}$ and $\mathrm{C}$ are uniformly distributed near the nozzle, then the mass flux of the precursor is of the order $C_{1} m_{11} v_{\text {in }} R_{1}^{2}$, where $C_{1}$ is the molecular concentration of Ta, $m_{11}$ is the mass of one Ta atom, $v_{\text {in }}$ is the gas velocity at the inlet boundary and $R_{1}$ is the nozzle radius (input of carbon to the mass flux would be of the same order). Our estimations show that the mass flux of the precursor is of the order of $10^{-5} \mathrm{~kg} \mathrm{~s}^{-1}$ for the considered concentrations of Ta and C (compare with $1.68 \times 10^{-3} \mathrm{~kg} \mathrm{~s}^{-1}$, which is the inlet mass flux of argon).

\section{Formation and growth of nanoparticles}

\subsection{Multi-component co-condensation}

We do not consider the chemical reactions that occur between the chemical species present in the system. In fact, one can expect over 100 reactions to occur (see, e.g. McFeaters et al 1994). Such analysis would be extremely difficult, if at all possible, and could not possibly be successfully combined with simulations of multi-dimensional gas flow. A different approach, called multi-component co-condensation (Shigeta and Watanabe 2007a, 2007b), was chosen to examine the formation of $\mathrm{TaC}$.

Following the phase diagram for the tantalum carbide system (see Los Alamos 1964, p 58), we can conclude that $\mathrm{TaC}$ does not exist in gaseous state. If $\mathrm{TaC}$, being in its liquid form, is heated above $2000{ }^{\circ} \mathrm{C}$ at atmospheric pressure, its vaporization starts with the loss of carbon and continues with evaporation of Ta (Los Alamos 1964, p 65), so two one-atomic gases form.

Let us summarize the scenario of formation of $\mathrm{TaC}$ nanoparticles from gaseous $\mathrm{Ta}$ and $\mathrm{C}$ that we utilize in this paper. By comparing the vapour pressures of $\mathrm{Ta}$ and $\mathrm{C}$ (see table A1 in appendix) we can conclude that Ta always nucleates first. We assume that, until the temperature becomes so low that the nucleation of Ta occurs, the chemical reaction between Ta and $\mathrm{C}$ does not happen. As soon as stable Ta clusters appear in the process of homogeneous nucleation, atoms of $\mathrm{C}$ and the rest of not-yet-nucleated Ta atoms precipitate on the clusters in the process of heterogeneous condensation. The particles collide and coalesce and are transported by the flow. Ta and
$\mathrm{C}$ form $\mathrm{TaC}$ compound, while being already in the liquid form within a particle.

Because of their very small size, the droplets solidify at significantly lower temperature than the bulk melting point of the solid (Friedlander 2000). This fact allows us to assume that the particles stay in the liquid form until their final deposition. This fact has two further important consequences. First, according to Shigeta and Watanabe (2007a, 2007b), we can make the conclusion about the chemical substance to be formed on the substrate based on the final composition of the particles. Second, the reduction in the melting point allows us to assume fast coalescence of two particles after their collision.

\subsection{Governing equations}

For the analysis of formation of the particles and their subsequent evolution, we have chosen the computational domain shown in figure 1( $a$ ) by a smaller (dark-grey) box. The formation and growth of nanoparticles should necessarily be considered in conjunction with their transport by the turbulent flow and with the turbulent transport and mixing of Ta and $\mathrm{C}$ gases. This is achieved following the approach used in the aerosol science. The fields of physical properties of the gas flow are taken from the first step of our study.

The particle composition is defined by two internal coordinates: $x_{1}$ and $x_{2}$ denoting the numbers of atoms of Ta and $\mathrm{C}$ in a particle, respectively. The particle density function, $n\left(\boldsymbol{r}, x_{1}, x_{2}\right)$, is introduced so that $n\left(\boldsymbol{r}, x_{1}, x_{2}\right) \mathrm{d} x_{1} \mathrm{~d} x_{2}$ stands for the concentration of particles containing the numbers of atoms of Ta and $\mathrm{C}$ in the ranges $\left[x_{1}, x_{1}+\mathrm{d} x_{1}\right]$ and $\left[x_{2}, x_{2}+\mathrm{d} x_{2}\right]$ and located at the physical coordinate (the external coordinate) $\boldsymbol{r}$. The general dynamic equation for the particle distribution function is (Friedlander 2000)

$$
\begin{aligned}
\frac{\partial n}{\partial t}+ & \nabla \cdot(n \boldsymbol{v})+\frac{\partial}{\partial x_{1}}\left[G_{1} n\right]+\frac{\partial}{\partial x_{2}}\left[G_{2} n\right]=\nabla \cdot\left(D_{\mathrm{t}} \nabla n\right)+J \\
& +\frac{1}{2} \int_{0}^{x_{1}} \int_{0}^{x_{2}} \mathrm{~d} \tilde{x}_{1} \mathrm{~d} \tilde{x}_{2} \beta\left(x_{1}-\tilde{x}_{1}, x_{2}-\tilde{x}_{2}, \tilde{x}_{1}, \tilde{x}_{2}\right) \tilde{n} \bar{n} \\
& -\int_{0}^{\infty} \int_{0}^{\infty} \mathrm{d} \tilde{x}_{1} \mathrm{~d} \tilde{x}_{2} \beta\left(x_{1}, x_{2}, \tilde{x}_{1}, \tilde{x}_{2}\right) n \tilde{n}
\end{aligned}
$$

Here $n \equiv n\left(x_{1}, x_{2}\right), \tilde{n} \equiv n\left(\tilde{x}_{1}, \tilde{x}_{2}\right)$ and $\bar{n} \equiv n\left(x_{1}-\tilde{x}_{1}, x_{2}-\tilde{x}_{2}\right)$. In equation (3), $J$ accounts for the birth of particles through the process of homogeneous nucleation, while the third and fourth terms in the left-hand side account for the growth of the particles by heterogeneous condensation. The integral terms in the right-hand side describe the coagulation of the particles due to collisions. $v$ in the second term in the left-hand side stands for the mean gas velocity (see figure $1(b)$ for the axial component, the radial component is almost zero). $D_{\mathrm{t}}$ is the coefficient of turbulent diffusion. The operator $\nabla$ stands for the differentiation along the external coordinates, $\nabla=\partial /(\partial \boldsymbol{r})$.

The processes of nucleation and condensation are going with strong consumption of $\mathrm{Ta}$ and $\mathrm{C}$ gases. The evolution of molecular concentrations of tantalum, $C_{1}$, and carbon, $C_{2}$, can 
(a)
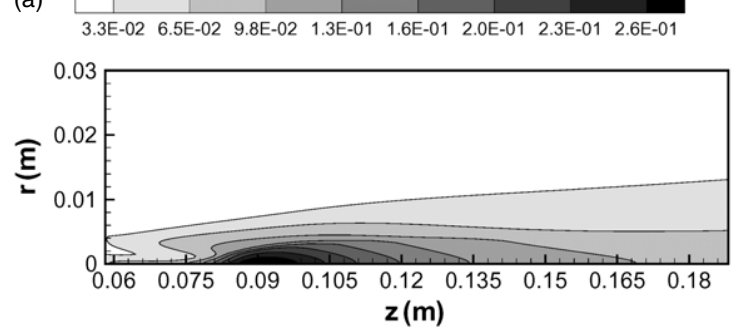

Figure 2. (a) Coefficient of turbulent diffusion $\mathrm{m}^{2} \mathrm{~s}^{-1}$. (b)

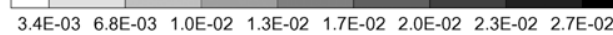

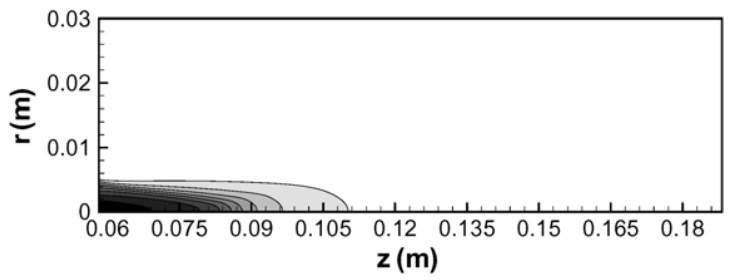

(b) Coefficient of molecular diffusion, $\mathrm{m}^{2} \mathrm{~s}^{-1}$ for Ta. be described by the following equations:

$$
\begin{aligned}
\frac{\partial C_{1}}{\partial t} & +\nabla \cdot\left(C_{1} \boldsymbol{v}\right)=\nabla \cdot\left(\left(D_{1}+D_{\mathrm{t}}\right) \nabla C_{1}\right)-x_{\mathrm{p}}^{*} J \\
& -\int_{0}^{\infty} \int_{0}^{\infty} \mathrm{d} x_{1} \mathrm{~d} x_{2}\left[G_{1} n\right],
\end{aligned}
$$

$$
\begin{aligned}
\frac{\partial C_{2}}{\partial t} & +\nabla \cdot\left(C_{2} v\right)=\nabla \cdot\left(\left(D_{2}+D_{\mathrm{t}}\right) \nabla C_{2}\right) \\
& -\int_{0}^{\infty} \int_{0}^{\infty} \mathrm{d} x_{1} \mathrm{~d} x_{2}\left[G_{2} n\right] .
\end{aligned}
$$

Here, $x_{\mathrm{p}}^{*}$ is the critical number of atoms in a nucleating particle discussed in the following section. In contrast to a single diffusion term considered in equation (3), two mechanisms for diffusion, molecular and turbulent, are taken into account in (4) and (5). $D_{1}$ and $D_{2}$ are the coefficients of molecular diffusion of Ta and $\mathrm{C}$, respectively. Our treatment of the two diffusion mechanisms can be justified by the assessment of their relative importance in the cases of particles and gases. The coefficient of turbulent diffusion is common for all three equations and determined from the simulations of the gas flow. This coefficient is defined as $D_{\mathrm{t}}=\mu_{\mathrm{t}} /\left(\rho \mathrm{S} c_{\mathrm{t}}\right)$, where $\mu_{\mathrm{t}}$ is the turbulent viscosity calculated by the $k-\epsilon$ model and $\mathrm{S} c_{\mathrm{t}}$ is the Schmidt number taken to be 0.7 (see, e.g. Launder and Spalding 1972). In order to estimate the coefficients of molecular diffusion we use the Stokes-Einstein expression (Friedlander 2000):

$$
D=\frac{k T}{f}=\frac{3 k T}{2 d_{1}^{2}}\left[\frac{1}{2 \pi p \rho}\right]^{1 / 2},
$$

where the friction coefficient $f$ is evaluated using the formula given by the kinetic theory with zero accommodation coefficient, $\rho$ is the density of the surrounding gas (figure $1(d)$ ), $p$ is the pressure (equal to atmospheric pressure with a good accuracy throughout the entire sub-domain) and $d_{1}$ is the diameter of a particle moving through the gas (in our case, it is either a molecule of Ta or $\mathrm{C}$, or a nanoparticle).

The computed fields of the turbulent diffusion coefficient and the molecular diffusion coefficient for Ta gas are plotted in figure 2. As one can see, the turbulent diffusivity is, in general, significantly larger. The molecular diffusivity can only become moderately important for the Ta and $\mathrm{C}$ gases in the area adjacent to the nozzle. For the nanoparticles, $D$ is significantly smaller because of the larger size $d_{1}$. Furthermore, as will be discussed below, the particles appear in the zone located substantially downstream from the nozzle, where the molecular diffusivity becomes very small and, thus, can be neglected.

The system of equations (3)-(5) needs to be complemented by the following conditions on the physical boundaries of the sub-domain:

Left boundary, $z=Z_{1}: \quad n=0$,

$C_{1}= \begin{cases}C_{10}, & \text { for } r \leqslant R_{\mathrm{in}}, \\ 0, & \text { for } r>R_{\mathrm{in}},\end{cases}$

$C_{2}= \begin{cases}C_{20}, & \text { for } r \leqslant R_{\mathrm{in}}, \\ 0, & \text { for } r>R_{\mathrm{in}},\end{cases}$

Axis of symmetry, $r=0: \frac{\partial n}{\partial r}=0, \quad \frac{\partial C_{1}}{\partial r}=0$,

$\frac{\partial C_{2}}{\partial r}=0$

Outer boundary, $r=R: \frac{\partial n}{\partial r}=0, \quad \frac{\partial C_{1}}{\partial r}=0$,

$\frac{\partial C_{2}}{\partial r}=0$

Outer boundary, $z=Z_{2}: \frac{\partial n}{\partial z}=0, \quad \frac{\partial C_{1}}{\partial z}=0$,

$\frac{\partial C_{2}}{\partial z}=0$

Here, $R, Z_{1}$ and $Z_{2}$ are the values of the radial and axial coordinates corresponding to the upper, left-hand and righthand boundaries of the dark-grey sub-domain in figure 1(a). As discussed before, the actual values of the Ta and $\mathrm{C}$ concentrations at the inlet boundary could not be determined with our approach. We assume that concentrations of both species are uniformly distributed along the inlet boundary at $r \leqslant R_{\text {in }}$, where $R_{\text {in }}=0.42 \mathrm{~cm}$ is chosen as a boundary of a zone with sufficiently high temperature so that no nucleation of the nanoparticles occurs outside the domain of investigation. The gun outlet includes an external cooling ring with the radius $R_{2}=0.53 \mathrm{~cm}$, which is larger than the radius $R_{1}=0.39 \mathrm{~cm}$ of the nozzle opening. This introduces additional complications by allowing the nucleation to be initiated within the cooling element close to the cooling walls. In reality, even if some nanoparticles are formed in this area, their number is insignificant because of the low concentrations of Ta and $\mathrm{C}$ gases transported there by the gas flow. In order to simplify this analysis we neglect these particles by introducing $R_{\text {in }}$ between 
$R_{1}$ and $R_{2}$ and prohibiting the nucleation of particles outside the computational domain.

The assumption about uniform distribution of species at the inlet boundary should not affect the results significantly due strong mixing of $\mathrm{Ta}$ and $\mathrm{C}$ species in the regions directly adhering to the inlet boundary.

The problem includes two additional internal coordinates for which we also need the boundary conditions:

$$
\begin{gathered}
x_{1}=0:\left[G_{1} n\right]=0, \quad x_{2}=0:\left[G_{2} n\right]=0, \\
x_{1}, x_{2} \rightarrow \infty: n \rightarrow 0 .
\end{gathered}
$$

These conditions mean zero flux at the boundaries of the solution domain in $\left(x_{1}, x_{2}\right)$. The boundary conditions at infinity are not needed for the solution of equation (3), but will be used for equations (4) and (5), as well as for deriving the simplified equations in section 4.6.

\subsection{Homogeneous nucleation}

The location and intensity of homogeneous nucleation is primarily determined by the value of the saturation vapour pressure, which strongly depends on temperature. As the hot jet coming out of the nozzle is cooled down by the surrounding air, the gas temperature drops by several thousand degrees within the distance of one or two centimetres (figure $1(c)$ ). As a result of that, the saturation pressure of Ta decreases below the partial pressure, and the homogeneous nucleation occurs.

There are numerous theoretical and semi-empirical models for the process of homogeneous nucleation, intented to predict the size of the stable nuclei and the nucleation rate. All these theories are based on the strong simplifying assumptions (such as spherical shape of the droplet, sizeindependent surface tension and steady-state nucleation) and able to give only qualitative predictions.

According to Girshick et al (1990), the rate of homogeneous nucleation is defined as

$$
J=2 C_{1} \frac{\sigma^{1 / 2} v_{11}}{\left(2 \pi m_{11}\right)^{1 / 2}} N_{0} \exp \left(-\frac{4 \Theta^{3}}{27\left(\ln \left(S_{1}\right)\right)^{2}}\right) .
$$

As a result of the nucleation, the stable droplets of the critical size containing $x_{\mathrm{p}}^{*}$ atoms of Ta are born, where

$$
x_{\mathrm{p}}^{*}=\left(\frac{2 \Theta}{3 \ln \left(S_{1}\right)}\right)^{3} .
$$

In the last two formulae, $\Theta=\sigma s_{11} /(k T)$ is the nondimensionalized coefficient of surface tension. $S_{1}=$ $C_{1} / C_{\mathrm{S}, 1}=p_{1} / p_{S, 1}$ is the oversaturation level, where $p_{1}$ is the partial pressure of the Ta gas, and $C_{\mathrm{S}, 1}$ and $p_{\mathrm{S}, 1}$ are the values of saturation concentration and pressure for Ta. $s_{11}, v_{11}$ and $m_{11}$ stand for the surface, volume and mass of one atom of Ta, respectively.

$N_{0}$ in (13) is the normalization factor, expression for which depends on the choice of the nucleation theory (see, e.g., Girshick et al 1990, Nadykto and Yu 2005). We accepted the expression of the so-called self-consistent model (Girshick et al 1990)

$$
N_{0}=C_{\mathrm{S}, 1} \exp \Theta,
$$

widely used in the literature. It has to be stressed that there is no consensus regarding the issue of appropriate expression for $N_{0}$. For instance, Nadykto and Yu (2005) argue that (15) gives too high values for the rate of nucleation and that a better agreement with the experiments can be obtained when $N_{0}$ is empirically taken as

$$
N_{0}=\frac{C_{\mathrm{S}, 1}}{S_{1}} \exp \Theta
$$

Finally, it is necessary to note that the formulae (13) and (14) assume that (i) the typical time for nucleation is much smaller than the typical time scale of evolution of particles and (ii) the critical nucleus consists of sufficiently many atoms, at least, more than 10. Our calculations show that the first assumption is justified. The nucleation can be modelled as a nearly instant process. On the other hand, the size of a critical nucleus given by (14) can become even smaller than the size of one atom. This is the typical result for the nucleation of materials with very low saturation vapour pressure (Friedlander 2000). In the calculations discussed below in this paper, all gas molecules of Ta are treated as stable nuclei if $x_{\mathrm{p}}^{*}$ becomes lower than 2 .

\subsection{Heterogeneous condensation}

As soon as stable droplets of Ta are formed, the heterogeneous condensation of Ta and $\mathrm{C}$ atoms starts to occur. The rate of the heterogeneous condensation is determined by the so-called growth law:

$$
G_{i}=\frac{\mathrm{d} x_{i}}{\mathrm{~d} t}, \quad i=1,2 .
$$

The expression for the rate of growth of a particle is derived based on the kinetic theory (Friedlander 2000):

$$
G_{i}=\frac{\alpha_{i} p_{S, i}}{\left(2 \pi m_{1 i} k T\right)^{1 / 2}}\left(S_{i}-1\right) \pi \mathrm{d}\left(x_{1}, x_{2}\right)^{2}, \quad i=1,2 .
$$

Here, indices $i=1$ and 2 refer to Ta and $\mathrm{C}$, respectively, $\alpha_{i}$ is the accommodation coefficients assumed to be 1 in this work, and $\mathrm{d}\left(x_{1}, x_{2}\right)=\left(d_{11}^{3} x_{1}+d_{12}^{3} x_{2}\right)^{1 / 3}$ is the particle diameter with $d_{11}$ and $d_{12}$ being the diameters of Ta and $\mathrm{C}$ atoms.

\subsection{Coagulations}

The inlet concentrations of the precursor used for our computations are sufficiently high for the growth of particles because of collisions and coagulations to be important. In fact, this mechanism can dominate growth due to heterogeneous condensation during the initial moments after the nucleation. We consider all collisions to be $100 \%$ effective and assume that the coalescence is rapid (because the particles are small), so the formation of fractal-like agglomerates does not occur.

The typical particles contain about 10-1000 atoms, and have the size of several nanometres. The collision frequency $N_{\text {col }}\left(x_{1}, x_{2}, \tilde{x}_{1}, \tilde{x}_{2}\right)=\beta\left(x_{1}, x_{2}, \tilde{x}_{1}, \tilde{x}_{2}\right) n\left(x_{1}, x_{2}\right) n\left(\tilde{x}_{1}, \tilde{x}_{2}\right)$, 
for such small particles is determined by the same formulae as the rate of collisions between molecules of a gas. The formula for the collision frequency function, $\beta$, is taken as the expression derived in the kinetic theory of gases (Friedlander 2000):

$$
\begin{aligned}
& \beta\left(x_{1}, x_{2}, \tilde{x}_{1}, \tilde{x}_{2}\right)=\left(\frac{3 k T}{\rho_{\mathrm{p}}}\right)^{1 / 2} \\
& \quad \times\left(\frac{1}{\mathrm{~d}\left(x_{1}, x_{2}\right)^{3}}+\frac{1}{\mathrm{~d}\left(\tilde{x}_{1}, \tilde{x}_{2}\right)^{3}}\right)^{1 / 2}\left(\mathrm{~d}\left(x_{1}, x_{2}\right)+\mathrm{d}\left(\tilde{x}_{1}, \tilde{x}_{2}\right)\right)^{2} .
\end{aligned}
$$

Here $\rho_{\mathrm{p}}$ is the density of the particles. This formula was derived for the case of collisions between atoms of one material. In our case, the particles differ from each other having different composition, i.e. different densities. This factor is not taken into account. Instead, (20) is used under the assumption that $\rho_{\mathrm{p}}$ is a constant equal to the density of $\mathrm{TaC}\left(13.9 \mathrm{~g} \mathrm{~cm}^{-3}\right)$. Formula (20) can be considered as only an estimate of the collision frequency. An additional simplifying assumption of constant density is unlikely to lower the accuracy of the estimate in any significant measure.

\subsection{Method of solution}

The system of equations and boundary conditions formulated in the previous section cannot be solved with the help of a direct numerical approach. The main difficulties, that lead to unrealistic computational requirements, arise due to the five dimensionality of the computational domain, which contains two external and two internal coordinates, and time. The equations need to be considerably simplified but in a way that retains the key physical effects.

We use the simplification conceptually similar to the method of moments (Friedlander 2000) but based on stronger assumptions. Validity of the approach will be verified through comparison with an exact solution of a model problem in section 5.1. One also needs to keep in mind that even a perfectly accurate solution of the full problem formulated above would only present a qualitative picture of the actual process due to approximate or even qualitative way in which the key factors, such as thermodynamic and transport coefficients, rate of homogeneous nucleation (13), growth laws (18) and the collision frequency function (20), can be determined.

The most important information for experiments and practical applications is the number concentration of particles, $N \equiv \int_{0}^{\infty} \int_{0}^{\infty} \mathrm{d} x_{1} \mathrm{~d} x_{2}(n)$, and their mean size, $\left(A_{1}+A_{2}\right) / N$ and composition, $A_{2} / A_{1}$. Here we introduce the first moments of the particle density function $n\left(\boldsymbol{r}, x_{1}, x_{2}\right), A_{1} \equiv$ $\int_{0}^{\infty} \int_{0}^{\infty} \mathrm{d} x_{1} \mathrm{~d} x_{2}\left(x_{1} n\right)$ and $A_{2} \equiv \int_{0}^{\infty} \int_{0}^{\infty} \mathrm{d} x_{1} \mathrm{~d} x_{2}\left(x_{2} n\right)$, which represent the mean values of the content of $\mathrm{Ta}$ and $\mathrm{C}$ in particles.

The equations for the zeroth and first moments of the particle distribution function can be easily obtained from the governing equation (3) and boundary conditions (11) and (12) and have the following form:

$$
\begin{aligned}
& \frac{\partial N}{\partial t}+\nabla \cdot(N \boldsymbol{v})=\nabla \cdot\left(D_{\mathrm{t}} \nabla N\right)+J\left(x_{\mathrm{p}}^{*}\right)+J_{\mathrm{col}}, \\
& \frac{\partial A_{1}}{\partial t}+\nabla \cdot\left(A_{1} v\right)-\int_{0}^{\infty} \int_{0}^{\infty} \mathrm{d} x_{1} \mathrm{~d} x_{2}\left[G_{1} n\right] \\
& =\nabla \cdot\left(D_{\mathrm{t}} \nabla A_{1}\right)+x_{\mathrm{p}}^{*} J\left(x_{\mathrm{p}}^{*}\right), \\
& \frac{\partial A_{2}}{\partial t}+\nabla \cdot\left(A_{2} \boldsymbol{v}\right)-\int_{0}^{\infty} \int_{0}^{\infty} \mathrm{d} x_{1} \mathrm{~d} x_{2}\left[G_{2} n\right]=\nabla \cdot\left(D_{\mathrm{t}} \nabla A_{2}\right) .
\end{aligned}
$$

Here $J_{\text {col }}$ is the term which represents the loss in the number of particles due to their coagulations.

In equations (21)-(23), the term $J_{\text {col }}$ and the integrals containing the growth laws, $G_{1}$ and $G_{2}$, cannot be computed on the basis of knowledge of $N, A_{1}$ and $A_{2}$ and, thus, have to be modelled. Let us assume for the beginning that the collisions can be neglected and consider the typical evolution of the particle density function $n\left(\boldsymbol{r}, x_{1}, x_{2}\right)$ taken for a fluid element moving with the flow. In principle, the size of the nucleating particles, $x_{\mathrm{p}}^{*}$, varies along the streamline following the variation of temperature and oversaturation $S_{1}$ (see (14)). In fact, however, as was found in our calculations of the nucleation rate (13), the rate has a strong peak at the location characterized by large oversaturation. As a result, the particles formed on a given streamline all have approximately equal size. The size then grows in the process of heterogeneous condensation. It can be seen from (18) that the rates of condensation of both Ta and $\mathrm{C}$ depend on the local thermodynamic conditions and the particle size and, so, should be approximately the same for all particles within a fluid element. The size and concentrations of $\mathrm{Ta}$ and $\mathrm{C}$ remain peaked around certain values. The evolution described above will be, of course, affected by the turbulent diffusion that would transport the particles and free Ta and C gases among the elements. One can still assume that, for any given physical location, the function $n\left(\boldsymbol{r}, x_{1}, x_{2}\right)$ continues to have a strong peak in the space of internal coordinates ${ }^{2}$.

We rely on this assumption in order to approximate the integrals in equations (22) and (23) as follows:

$$
\int_{0}^{\infty} \int_{0}^{\infty} \mathrm{d} x_{1} \mathrm{~d} x_{2}\left[G_{i} n\right]=N G_{i}^{*}, \quad i=1,2,
$$

where the growth laws are

$$
\begin{aligned}
G_{i}^{*} & =\frac{\alpha_{i} p_{S, i}}{\left(2 \pi m_{1 i} k T\right)^{1 / 2}}\left(S_{i}-1\right) \pi\left(\frac{A_{1}}{N} d_{11}^{3}+\frac{A_{2}}{N} d_{12}^{3}\right)^{2 / 3}, \\
& i=1,2
\end{aligned}
$$

i.e. the numbers of atoms of $\mathrm{Ta}$ and $\mathrm{C}$ in a particle are approximated by the average values $x_{1} \approx A_{1} / N$ and $x_{2} \approx A_{2} / N$.

Let us return to the effect of collisions between the particles, which is important for the values of concentrations interesting for our study $\left(C_{10}>10^{19} \mathrm{~m}^{-3}\right)$. The coagulations

2 Of course, one has to expect that the values of $x_{1}$ and $x_{2}$, at which the peak is observed would vary greatly with the location $\boldsymbol{r}$. The expectations will be fully supported by our results in section 5.2. 
widen the peak in the particle density distribution, which renders the assumption of nearly monodisperse distribution at a given space location less grounded. Understanding that it would negatively affect the quality of the prediction, but having no feasible alternative in the way of direct simulations, we choose to extend the assumption to the case when the collisions of particles are taken into account. As we will see below, this assumption works reasonably well, at least, for the initial moments after the nucleation (when the majority of collisions occurs) for chemical substances with low vapour pressure points, such as Ta. The collision term $J_{\text {col }}$ is modelled in the manner similar to (24) and (25):

$$
J_{\mathrm{col}}=-\frac{1}{2} \beta N^{2}, \quad \beta=\left(\frac{3 k T}{\rho_{\mathrm{p}}}\right)^{1 / 2}\left(\frac{A_{1}+A_{2}}{N}\right)^{1 / 6} \text {. }
$$

The assumption of nearly monodisperse distribution of particles underlying the approximations (24)-(26) is quite strong and should be used with full understanding of the limitations it imposes on the type of the systems that can be considered. In particular, neglecting the effect of the turbulent diffusion on the dispersions of particle in internal coordinate is only possible if the typical time of residence of particles within the system is smaller than or, at least, comparable to the typical time of turbulent diffusion. The latter can be estimated as $L^{2} / D$, where $L$ and $D$ are the typical length scale of variation of the internal particle properties and the typical turbulent diffusion coefficient, respectively. Using $L=0.01 \mathrm{~m}$ and $D=0.1 \mathrm{~m}^{2} \mathrm{~s}^{-1}$ (see the results of calculations in section 5.2), we obtain the diffusion time of about $10^{-3} \mathrm{~s}$. The residence time can be estimated as between $5 \times 10^{-4} \mathrm{~s}$ and $10^{-3} \mathrm{~s}$. Somewhat shorter time is obtained if one estimates the duration of the period, during which the formation of particles (nucleation, condensation, and most of coagulations) is virtually complete (see sections 5.1 and 5.2). We see that the requirement is satisfied, albeit barely, for our system. This would not, however, be true for some other systems of synthesis of nanoparticles characterized by significantly longer residence times.

\section{Results}

\subsection{One-dimensional one-component calculations: verification of the simplified approach}

To check the validity of the assumptions made in the preceding section we need some results of direct analysis. Such results are impossible to obtain for the full system (3)-(5) in five dimensions with computationally extremely expensive evaluation of the collision term. For this reason, we apply the direct approach to a model system, in which the number of dimensions is reduced. First, we completely neglect the effect of turbulent diffusion. This allows us to consider only one space coordinate taken along a streamline, instead of two coordinates and time. Furthermore, we assume that only Ta gas participates in the particle formation, thus reducing the number of internal coordinates to one. The resulting one-dimensional one-component problem can be solved directly and fully, with retention of transport, nucleation, condensation, and collision effects for the particles and transport and consumption for the Ta gas. The purpose of the analysis is two-fold. First, we seek to verify the simplifying assumptions leading to (24)-(26). Second, the direct solution of the low-dimensional system provides insight into the key features of the process of particle formation.

The low-dimensional problem is solved using the finitedifference method. The continuous internal coordinate, $x_{1}$, is replaced by the discrete coordinate with a step corresponding to one atom. The maximum possible number of Ta atoms in a particle is assumed to be 8000 . The accuracy of the calculations is controlled through the conservation law $C_{1}+A_{1}=C_{10}$.

The results are plotted in figure 3 together with the analogous curves (in figures $3(c)$ and $(d)$ obtained with the help of the simplified physical model proposed in the previous section. Two initial concentrations of Ta atoms are examined, namely $C_{10}=1 \times 10^{21} \mathrm{~m}^{-3}$ and $C_{10}=5 \times 10^{21} \mathrm{~m}^{-3}$. The streamlines are obtained by integration of the velocity field along two representative lines, corresponding to the initial coordinates $\left(z=Z_{1}, r=0\right)$ and $\left(z=Z_{1}, r=R_{\text {in }}\right.$ ) (see figure $1(a)$ and boundary conditions (7)). The calculations are carried out within the sub-domain shown in figure 1(a), and are terminated when the fluid element reaches one of the boundaries. The evolution of the fluid elements along the representative streamlines lasts $t_{\mathrm{f}}=7.5 \times 10^{-4} \mathrm{~s}$ and $t_{f}=8.9 \times 10^{-4} \mathrm{~s}$, respectively.

The computed fields are shown in figure 3 as function of the time of movement from the beginning of the streamline to a given location, in other words, for a given fluid element. The values of temperature along the streamlines (figure 3(a)) differ at the initial moments in accordance with the sharp temperature gradient near the nozzle seen in figure $1(c)$. The difference becomes much smaller farther downstream and the nucleation, which approximately corresponds to temperature of $3500 \mathrm{~K}$, occurs at very close moments of time. As a result, the curves for the number and average size of the particles calculated for the two streamlines show very similar behaviour. Only the data obtained for the streamline corresponding to the movement along the symmetry axis (initial point $\left(Z_{1}, 0\right)$ ) are shown in figures $3(b)-(d)$.

Figure $3(b)$ shows the nucleation rate, $J$, and the size of stable nuclei, $x_{\mathrm{p}}^{*}$, obtained by solution of one-dimensional onecomponent problem. According to the theory of homogeneous nucleation, atoms of a gas always form clusters, which become stable nuclei if their size $x_{\mathrm{p}}$ exceeds $x_{\mathrm{p}}^{*}$ given by (14). In our calculations, at the initial moments, while temperature is high and no nucleation occurs, $x_{\mathrm{p}}^{*}$ is set as the maximum possible size of a particle, 8000. At a later stage, when the oversaturation $S_{1}$ becomes larger than $1, x_{\mathrm{p}}^{*}$ decreases rapidly and the stable nuclei appear. At some stage of the nucleation process, $x_{\mathrm{p}}^{*}$ becomes less than 2 . The situation changes dramatically since, after this moment, any free atom of Ta should be considered as a nucleus. This is reproduced in the computations by replacing all free, not-yet-nucleated, atoms of Ta by the same number of stable nuclei with the size of 1 atom. At this moment, the nucleation rate $J$ drops to 0 as no gas for nucleation is left, and $x_{\mathrm{p}}^{*}$ returns to 8000 . 

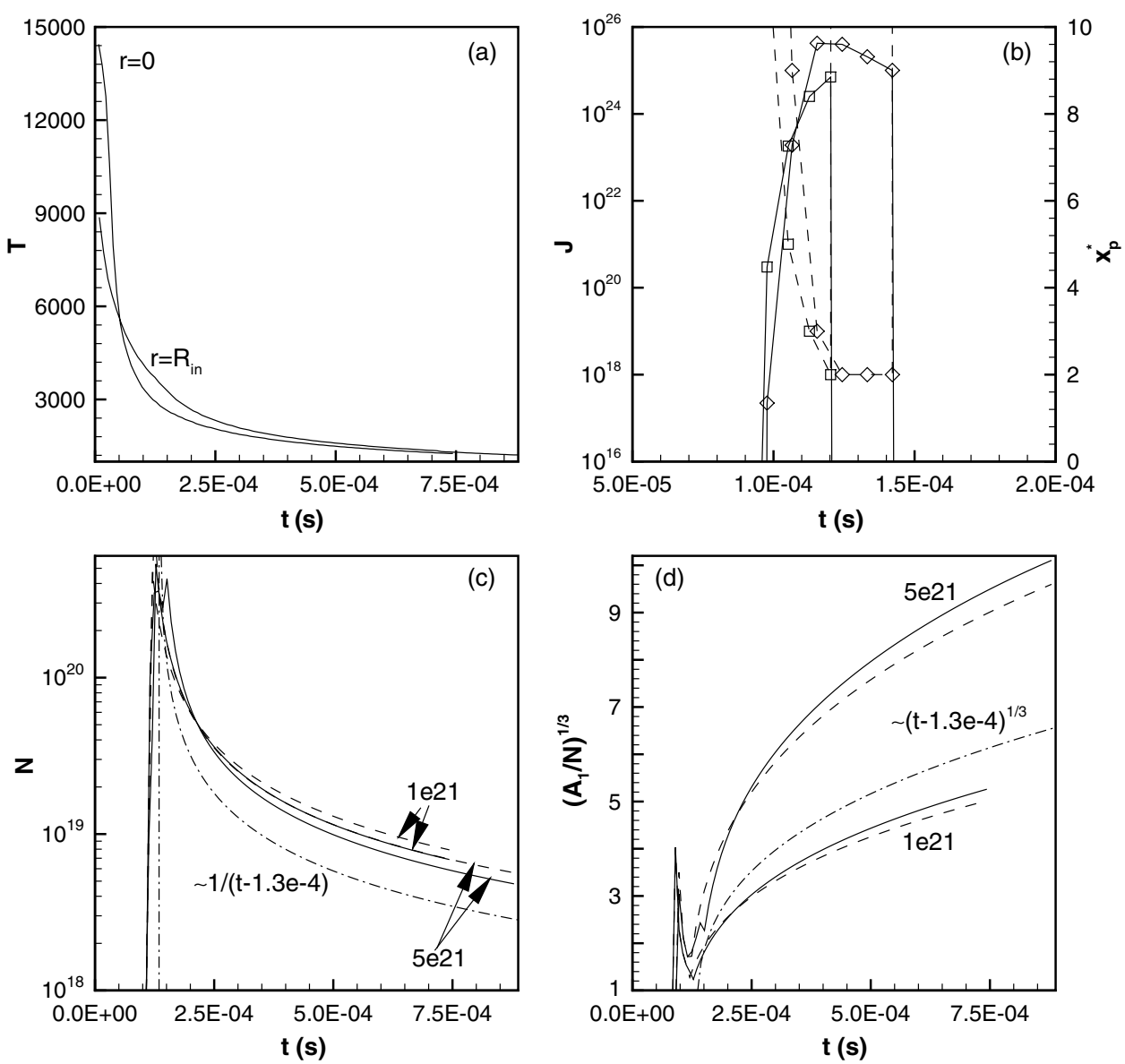

Figure 3. One-dimensional one-component problem. Results of direct solution and their comparison with the results of the simplified model. (a) Temperature (K) along the two streamlines with initial coordinates $\left(Z_{1}, 0\right)$ and $\left(Z_{1}, R_{\text {in }}\right)$. $(b)$ Rate of homogeneous nucleation, $J$ $\left(\mathrm{m}^{3} \mathrm{~s}^{-1}\right)(-)$ and the size of stable nuclei, $x_{\mathrm{p}}^{*},(---)$ obtained in direct calculations. The values of $x_{\mathrm{p}}^{*}$ are the integer approximations of (14). $\square$ corresponds to inlet concentration of Ta $C_{10}=1 \times 10^{21} \mathrm{~m}^{-3}, \diamond$ to $C_{10}=5 \times 10^{21} \mathrm{~m}^{-3}$. (c) Number concentration of particles $\left(\mathrm{m}^{-3}\right)$; the solid lines correspond to direct calculations, the dashed lines to the simplified model. Results for two initial concentrations of Ta $C_{10}=1 \times 10^{21} \mathrm{~m}^{-3}$ and $C_{10}=5 \times 10^{21} \mathrm{~m}^{-3}$ are shown as marked. $(d)$ Mean size of particles, the lines are as in $(c)$. The results in $(b)-(d)$ are obtained for the streamline with the initial point $\left(Z_{1}, 0\right)$. The dash-dotted lines in $(c)$ and $(d)$ correspond to the asymptotic solution $(28)$, in which $1.3 \times 10^{-4} \mathrm{~s}$ is taken as an approximation of the nucleation point.

The total number of particles and the average number of Ta atoms in a particle are plotted in figures $3(c)$ and $(d)$, respectively. The factor $\left(A_{1} / N\right)^{1 / 3}$ in figure $3(d)$ needs to be multiplied by the size of one molecule of Ta, i.e. $d_{11}$, to give the average size of the particle. One can see in figures $3(c)$ and $(d)$ that the disagreement between the curves obtained using the direct computation and the simplified model of section 4.6 is small, less than $15 \%$ for $N$ and less than $5 \%$ for $\left(A_{1} / N\right)^{1 / 3}$. Similar results were obtained for other physical quantities, streamlines and initial parameters considered. The accuracy of the simplified physical model in prediction of the particle nucleation and growth by condensation and collisions is satisfactory. The model can be considered a legitimate approach and used for solution of multi-dimensional multicomponent problems. Some comments can be made in regard the assumptions underlying the model. During the short period of time following the initial moment of nucleation, the particles are nearly monodisperse, primarily due to the fact that, at some moment, because of the very low vapour pressure, all molecules of Ta become stable nuclei having the same size of 1 atom, and approximations (24)-(26) are reasonably accurate.
The following condensation does not change the monodisperse character of the distribution significantly since all particles within the same fluid element grow at approximately equal rate. On the other hand, coagulations lead to widening the peak of the particle density function, presumably rendering the simplifying assumptions leading to (24)-(26) less valid. The negative effect is, however, offset by the fact that the number of particles $N$ decreases because of coagulations. Since the number of collisions is proportional to the square of the number of particles, the impact of the collision term diminishes, and so does the error associated with modelling of this term.

At every moment of the particle evolution, the conservation law $C_{1}+A_{1}=C_{10}$ is to be satisfied. Our results also show that the condensation of Ta gas on the particles occurs very rapidly mostly because of low saturation vapour pressure of Ta. As a result, the large part of the system evolution (after $t \geqslant 1.5 \times 10^{-4} \mathrm{~s}$ ) is characterized by complete condensation of Ta. The conservation law leads to particularly simple behaviour in this asymptotic state. The amount of condensed material is given by $A_{1}=C_{10}$ and the average size of the particle is approximately $C_{10} / N$. 
(a) $0.0 \mathrm{E}+00 \quad 7.1 \mathrm{E}+20 \quad 1.4 \mathrm{E}+21 \quad 2.1 \mathrm{E}+21 \quad 2.9 \mathrm{E}+21 \quad 3.6 \mathrm{E}+21 \quad 4.3 \mathrm{E}+21 \quad 5.0 \mathrm{E}+2$

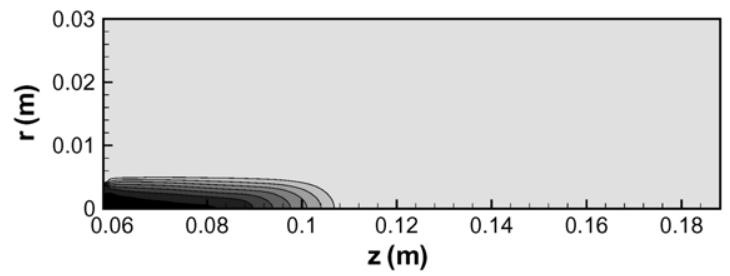

(c)

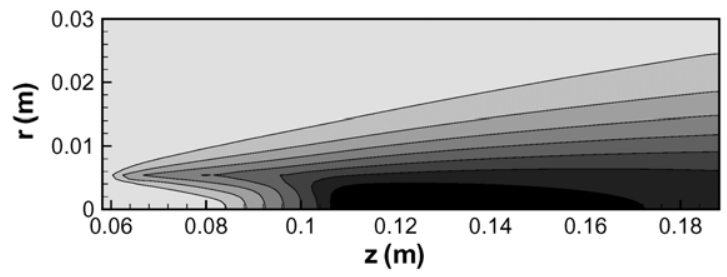

(e)

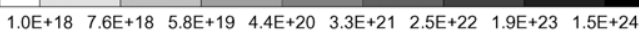

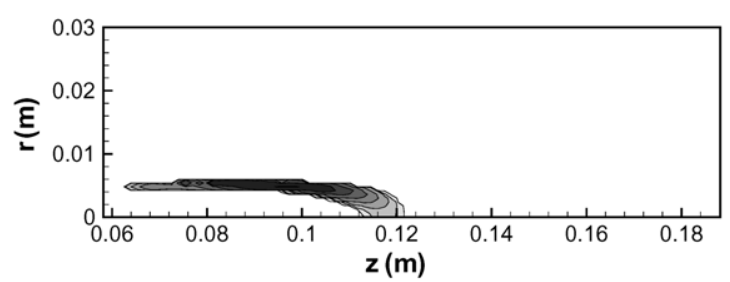

(b)
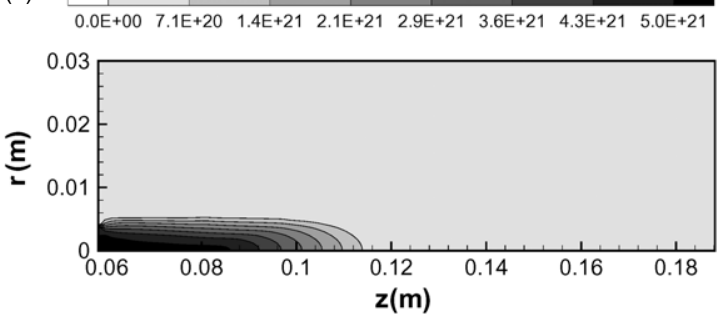

(d)

$0.0 \mathrm{E}+00 \quad 7.1 \mathrm{E}+20 \quad 1.4 \mathrm{E}+21 \quad 2.1 \mathrm{E}+21 \quad 2.9 \mathrm{E}+21 \quad 3.6 \mathrm{E}+21 \quad 4.3 \mathrm{E}+21 \quad 5.0 \mathrm{E}+21$

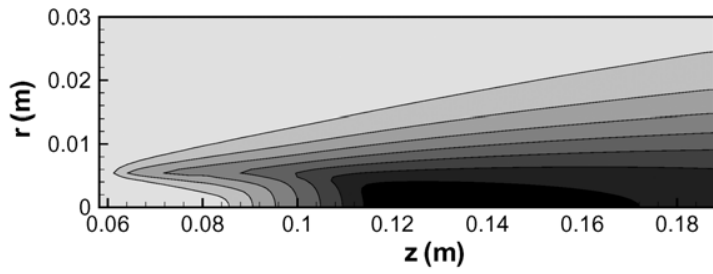

(f) \begin{tabular}{llllllll}
\hline & & & & & & \\
\hline $1.0 \mathrm{E}+18$ & $2.5 \mathrm{E}+18$ & $6.0 \mathrm{E}+18$ & $1.5 \mathrm{E}+19$ & $3.7 \mathrm{E}+19$ & $9.0 \mathrm{E}+19$ & $2.2 \mathrm{E}+20$ & $5.4 \mathrm{E}+20$
\end{tabular}

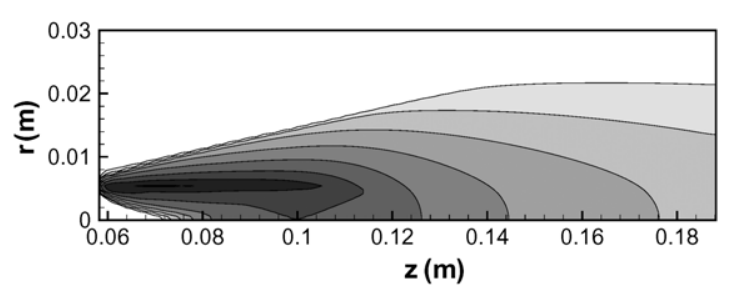

Figure 4. 2D calculations. The results are obtained for $C_{10}=C_{20}=5 \times 10^{21} \mathrm{~m}^{-3}$. (a) Concentration of free Ta (m $\mathrm{m}^{-3}$ ) atoms $C_{1}$. (b) Concentration of free $\mathrm{C}\left(\mathrm{m}^{-3}\right)$ atoms $C_{2}$. (c) Concentration of Ta $\left(\mathrm{m}^{-3}\right)$ in condensed state $A_{1}$. $(d)$ Concentration of $\mathrm{C}\left(\mathrm{m}^{-3}\right)$ in condensed state $A_{2}$. (e) Nucleation rate $J\left(\mathrm{~m}^{-3} \mathrm{~s}^{-1}\right)$. ( $f$ ) Concentration of particles $N\left(\mathrm{~m}^{-3}\right)$.

We can derive an asymptotic solution by observing that, after the nucleation process has ended, the equation for the number concentration of particles in a fluid element becomes

$$
\frac{\mathrm{d} N}{\mathrm{~d} t}=-\frac{1}{2} \beta N^{2}
$$

The collision frequency function, $\beta$, depends on $t$ through temperature and particle size. If, however, we additionally assume that this dependence is weak (decrease in the temperature is compensated by the particle growth), then it is easy to obtain the solution:

$$
N=\frac{2 / \beta}{t-\left(t_{0}-\frac{2}{\beta N_{0}}\right)}=\frac{2 / \beta}{t-t_{n}},
$$

where $N_{0}$ is the initial number of nuclei and $t_{0}$ is the point of nucleation. It can be shown that $\beta N_{0} t_{0} \gg 1$, which means $t_{n} \approx t_{0}$.

To verify the asymptotic solution, we plot curve (28) and the corresponding curve for $\left(A_{1} / N\right)^{1 / 3}$ in figures $3(c)$ and $(d)$, respectively. The time moment $t=1.3 \times 10^{-4} \mathrm{~s}$ is taken as an approximation of $t_{n}$. It can be seen that the solution follows the asymptotic behaviour closely.

\subsection{Two-dimensional results: formation of TaC nanoparticles}

We now would like to apply the simplified physical model verified in the previous section to study the formation of particles in more realistic situations. The calculations are carried out within the computational region with the horizontal and vertical sizes $\left(Z_{2}-Z_{1}\right) \times R=13 \times 3 \mathrm{~cm}^{2}$. This region is shown in figure $1(a)$ as a dark-grey sub-domain. Equations (21)-(23) are written in cylindrical coordinates and discretized with the help of the finite-difference scheme of the second order. The numerical resolution is $n_{z} \times n_{r}=$ $450 \times 100$ points on a uniform grid. The steady-state solution of equations (21)-(23) is determined by using the relaxation method.

The results of simulations for the case of equal inlet concentrations of Ta and C, $C_{10}=C_{20}=5 \times 10^{21} \mathrm{~m}^{-3}$, are shown in figure 4 . As one can see, high concentrations of free Ta and C gases, $C_{1}$ and $C_{2}$, (figures $4(a)$ and $(b)$ ) are only found in the region adjoining to the nozzle. The gases are rapidly consumed by the particles and are nearly absent outside this area. This feature of the 2D solution is in agreement with the results obtained in the previous section for the simplified one-dimensional problem. Figures $4(c)$ and $(d)$ show the amount of material in the condensed form $\left(A_{1}\right.$ and $\left.A_{2}\right)$. It is interesting to note that although these characteristics become virtually uniform in the streamwise direction outside the area of nucleation and intensive condensation, they retain strong variation across the streamlines. This is the main reason of only marginal validity of the assumption of weak effect of turbulent diffusion of particles used to derive the simplified model in section 4.6. On the other hand, we now see an additional argument supporting the validity as illustrated in figure $2(a)$. 


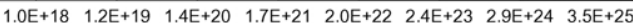

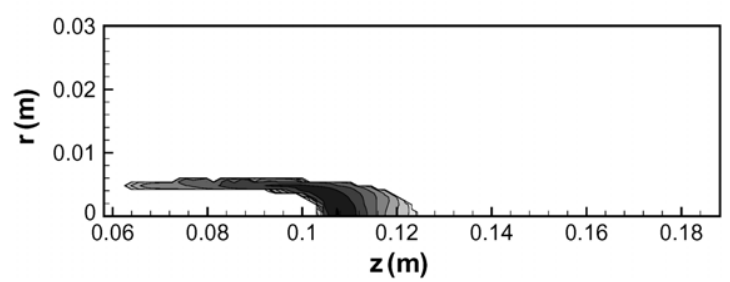

Figure 5. 2D calculations with artificially reduced turbulent diffusivity. (a) Nucleation rate $J\left(\mathrm{~m}^{-3} \mathrm{~s}^{-1}\right)$. (b) Concentration of particles $N$ $\left(\mathrm{m}^{-3}\right)$. The results are shown for $C_{10}=C_{20}=5 \times 10^{21} \mathrm{~m}^{-3}$.

The diffusion coefficient is relatively small in the areas, where strong cross-stream gradients of $A_{1}$ and $A_{2}$ are observed. The concentration of particles, $N$ shown in figure $4(f)$ decreases in the streamwise direction due to particle collisions. Here, again, the diffusion in the physical space may be of importance.

The isolevels of the particle nucleation rate $J$ given by (13) are plotted in figure 4(e). The picture illustrates a striking effect, which is essentially $2 \mathrm{D}$ and could not be detected in one-dimensional simulations along a streamline such as those conducted by Shigeta and Watanabe (2007a, 2007b). The nucleation mostly occurs in the lateral area of the jet despite the fact that the concentration of incoming Ta gas, $C_{1}$, is much higher in the central area (see figure 4(a)). This can be explained by the presence of cross-stream turbulent diffusion and the rapid decrease of temperature in the lateral area due to mixing with cold air (see figure 1(c)). High supersaturation level $S_{1}$, and, thus, conditions for intensive nucleation appear in the lateral area at a smaller value of $z$ than in the core area. In the result of nucleation, the concentration of free Ta in the lateral area decreases as shown in figure 4(a). Strong crossstream gradient of $C_{1}$ leads to strong cross-stream turbulent diffusive transport. In fact, most of $C_{1}$ in the central area is depleted before the conditions for nucleation are ripe, as can be seen by comparing figures $4(a)$ and $(e)$. The effect of turbulent diffusion is further illustrated in figure 5 where the nucleation rate and the concentration of particles are shown for the calculations, in which the turbulent diffusivity is artificially reduced tenfold. One can see that the position of the maximum nucleation rate is shifted toward the centre of the jet, where it reaches a much higher value than in figure 4(e). Figure 5(b) shows how the reduction in diffusivity influences the spatial distribution and number of nucleated particles.

Figure 6 shows the mean size of particles and their mean composition defined as $A_{2} / A_{1}$ for three runs with different inlet parameters: $C_{10}=C_{20}=5 \times 10^{21} \mathrm{~m}^{-3}$, $C_{10}=C_{20}=1 \times 10^{21} \mathrm{~m}^{-3}$, and $C_{10}=1 \times 10^{21} \mathrm{~m}^{-3}$, $C_{20}=5 \times 10^{21} \mathrm{~m}^{-3}$. As one can see, the consumption of gases through both nucleation and condensation is sufficiently fast, so that the chemical composition of particles remains unchanged for the main part of their trajectories, while their size grows by coagulations. The diffusion is quite important in the area of nucleation. Its strength, however, decreases downstream, which reduces its effect on the particle behaviour. Downstream, the particles move predominantly along the streamlines generated by the mean velocity field. The final size of the particles is approximately $3.3 \mathrm{~nm}, 1.7 \mathrm{~nm}$ and $2.7 \mathrm{~nm}$ for the first, second and third run, respectively. The final particle composition is, almost exactly, 1 in the first and second cases and 5 in the third case, which corresponds to the ratios of initial concentrations of $\mathrm{Ta}$ and $\mathrm{C}$, and illustrates the effect of complete rapid consumption of the initially injected gases.

As discussed in section 4.3, expression (15) for the normalization factor of the nucleation rate (13) is not universally accepted. In order to investigate the possible effect of the choice of model for $N_{0}$, we repeat the computations for $C_{10}=C_{20}=5 \times 10^{21} \mathrm{~m}^{-3}$ with the alternative definition (16). The results are shown in figure 7. Comparing figures $7(a)$ and 4(e), we see that the maximum nucleation rate given by (16) and (13) is, indeed, more than three orders of magnitude lower than the rate predicted by (15) and (13). It is also characterized by somewhat stronger lateral distribution. Quite remarkably, however, the modification of the nucleation process does not lead to any significant change of the concentration of particles (figures $7(b)$ and $4(f)$ ), their mean diameter (figures $7(c)$ and $6(a)$ ) and chemical composition (figures $7(d)$ and $6(b)$ ). The explanation is that the majority of particles in all considered cases are born when the critical size of a stable nucleus $x_{\mathrm{p}}^{*}$ becomes smaller than 2 so all free atoms are considered as nuclei. As a result, the total number of nucleating particles is only partially determined by the form of the expression for the nucleation rate. The following evolution is dominated by heterogeneous condensation (briefly) and by collisions, and, thus, is insensitive to the nucleation rate. Overall, the expression for the nucleation rate has only a slight influence on the total number of particles, their size and composition, if the nucleation of the gas with low vapour pressure (e.g. Ta) is examined.

\section{Concluding remarks}

In this paper we pursued two main goals. First, we wanted to develop a model that would allow us to analyse the formation of multi-component nanoparticles. The approach based on the general dynamic equation (3) and models for homogeneous nucleation, heterogeneous condensation, and particle collision was chosen. The general dynamic equation is, by itself, not new. It has been used in the past to describe the aerosol formation (Friedlander 2000) and other processes. Several methods of solution were developed including those based on the use of the first few statistical moments. Following this method, the equation is rewritten as a set of equations for the several low moments of the particle density function. This set 
(a)

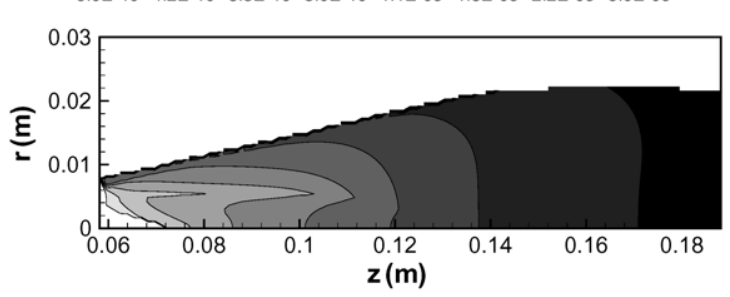

(c)

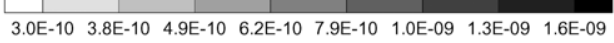

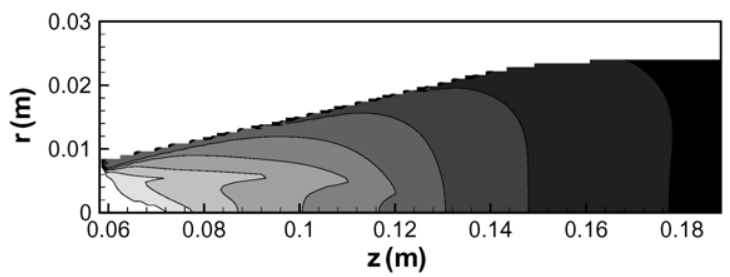

(e)
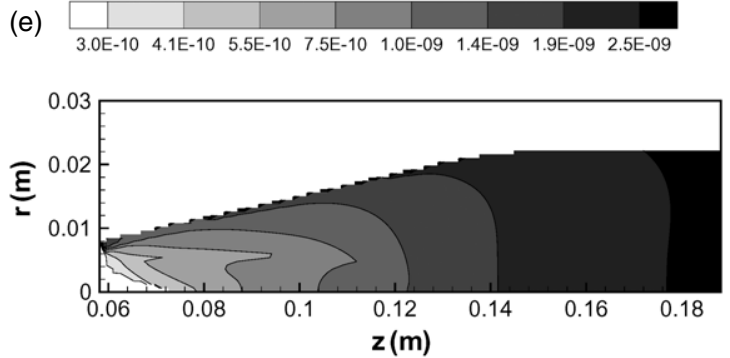

(b)

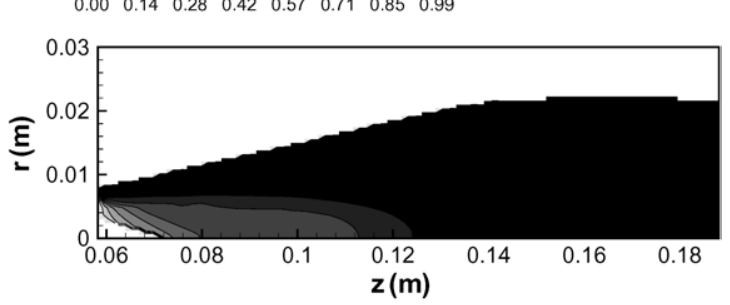

(d)
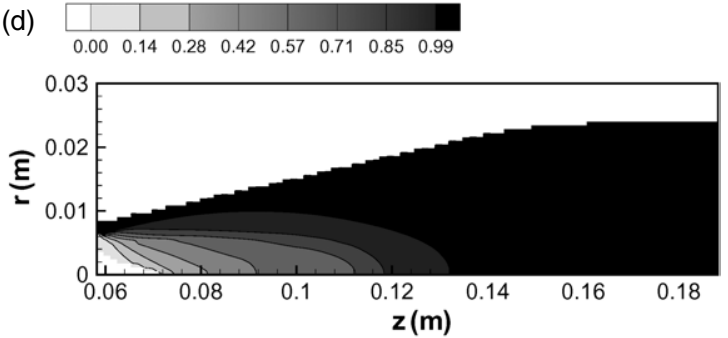

(f)
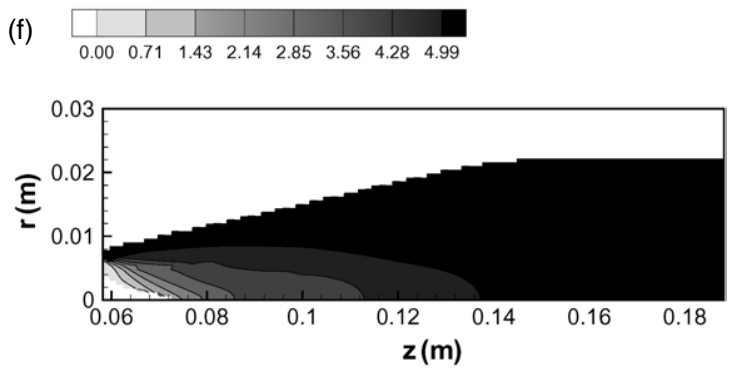

Figure 6. 2D calculations. $(a),(c)$ and $(e)$ : mean diameter of particles $(\mathrm{m}) .(b),(d)$ and $(f)$ : mean composition of particles. The results are shown for the inlet concentrations $C_{10}=C_{20}=5 \times 10^{21} \mathrm{~m}^{-3}(a)$ and $(b), C_{10}=C_{20}=1 \times 10^{21} \mathrm{~m}^{-3}(c)$ and $(d)$, and $C_{10}=1 \times 10^{21} \mathrm{~m}^{-3}$ and $C_{20}=5 \times 10^{21} \mathrm{~m}^{-3}(e)$ and $(f)$. The data are shown only for the region with particle concentration $N>1 \times 10^{18} \mathrm{~m}^{-3}$.

(a)
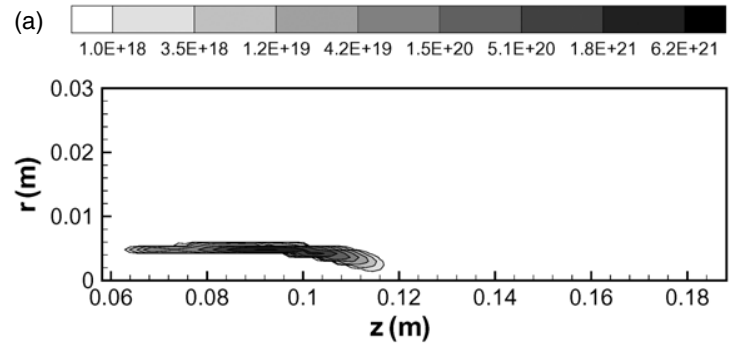

(c)

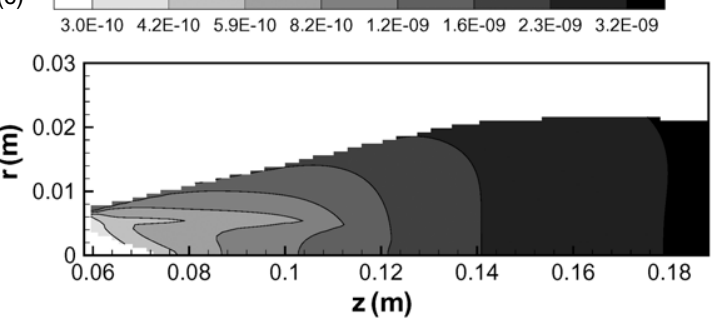

(b)
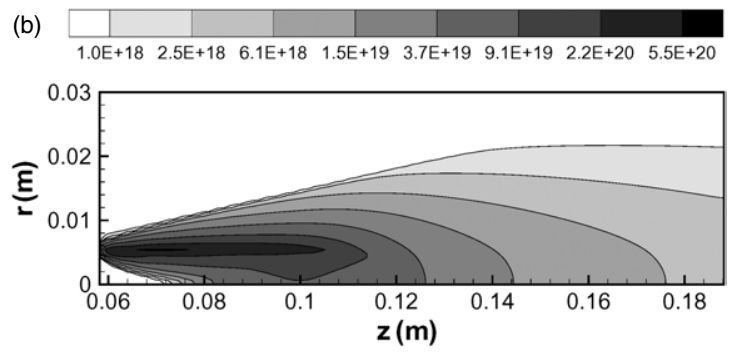

(d)
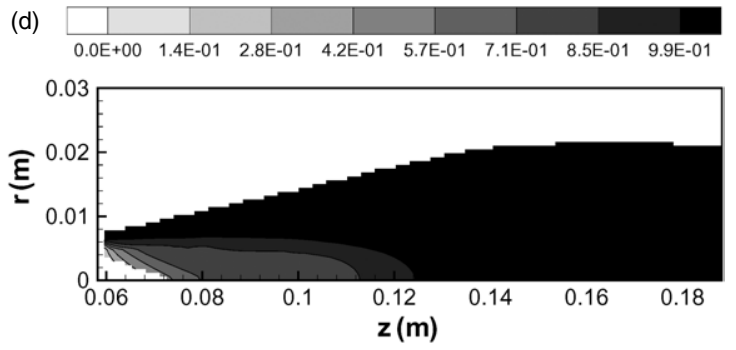

Figure 7. 2D calculations with the nucleation rate defined by (16). (a) Nucleation rate $J\left(\mathrm{~m}^{-3} \mathrm{~s}^{-1}\right)$. $(b) \mathrm{Concentration}$ of particles $N\left(\mathrm{~m}^{-3}\right)$. (c) Mean diameter of particles. (d) Mean composition of particles. The results are shown for the initial concentrations $C_{10}=C_{20}=5 \times 10^{21} \mathrm{~m}^{-3}$. $(c)$ and $(d)$ show only the region with particle concentration $N>1 \times 10^{18} \mathrm{~m}^{-3}$.

of new equations is closed via the use of additional assumptions or approximations. For example, log-normal distribution was assumed for particle density function in some studies, while quadrature evaluation of integrals was employed in others. This approach considerably reduces the number of degrees of freedom of the system and makes numerical treatment feasible even for complex configurations. The method of moments has, however, been developed for analysis of onecomponent particles, when the particle density function is the function of only one internal parameter, e.g. particle size. 
Table A1. Vapour pressure reference points. The data are taken from Stull (1972) and Leider et al (1973).

\begin{tabular}{lllllllll}
\hline & $p(\mathrm{~Pa})$ & 1 & 10 & $10^{2}$ & $10^{3}$ & $10^{4}$ & $10^{5}$ & $p_{\text {atm }}$ \\
\hline Carbon: & $T(\mathrm{~K})$ & - & 2839 & 3048 & 3289 & 3572 & 3908 & - \\
Tantalum: & $T(\mathrm{~K})$ & 3297 & 3597 & 3957 & 4395 & 4939 & 5634 & 5731 \\
\hline
\end{tabular}

If the particles are compounds of two chemical elements and their composition is as important as the size, additional internal coordinates are required. The key result of this paper is development and verification of the method for efficient numerical solution of the general dynamic equation in the case of formation of multi-component nanoparticles in a thermal plasma jet.

Our second result is the application of the method for numerical analysis of co-condensation of TaC nanoparticles in a dc plasma reactor. The analysis allows us to investigate the process of synthesis and reach conclusions about the importance of various physical mechanisms for the final distribution of the size and chemical composition of the particles. For instance, the one-dimensional analysis along the streamlines is shown to be insufficient. Introduction of the streamlines reduces the physical dimensionality of the problem, but it does not take into account the molecular, and, more importantly, turbulent diffusion. Owing to high velocities of the gas in the dc reactors, the jet flow is turbulent, which results in particularly strong turbulent mixing at the lateral boundaries of the jet. Even moderate turbulent diffusion present in our calculations leads to perceptible difference in the location of the main zone of particle nucleation (as well as in the predominant nucleus size and nucleation rates, both of which are the functions of the local temperature). The turbulent diffusion is also important at later stages of the evolution of particles, where it affects their spacial distribution. It is necessary to add that in a real experiment one can expect even stronger turbulent mixing than that present in our simulations, because of additional disturbances introduced, e.g. by the injection of a precursor.

An advantage of our approach is that no terms in the general dynamic equation are omitted. This allows us to analyse the relative importance of various physical effects for the synthesis process. In particular, we found that the effect of coagulation cannot be neglected since it is of, at least, equal importance as the effects of nucleation and condensation during the earlier stages of the particle evolution and dominate at later stages. We found that this observation remains valid even for low initial concentration of the precursor. The reason is the very low vapour pressure of Ta gas. At some moment, all gas molecules of Ta become stable nuclei and so the total number of particles available for collisions does not decrease significantly.

Our model renders the numerical consideration feasible at the cost of introducing additional inaccuracy into the results. Nevertheless, we would like to stress that this source of inaccuracy is equal to or less significant than the sources inevitably present due to inaccurate or outright qualitative character of the physical models used for different aspects of the process. Of course, each of them affects the quality of the prediction of the final particle distribution to a different degree. For example, we found that the effect of the choice of nucleation model is quite weak, as the majority of nuclei appear at the moment when the critical cluster size reaches the size of one atom. We conclude this part of the discussion saying that, due to the inevitable uncertainty, the model presented in this paper provides only a qualitative prediction, although based on the consideration of the key physical aspects of the process.

In this paper we do not consider the deposition of the particles on the target substrate. The size of the computation domain in the $z$-direction is chosen to be $\left(Z_{2}-Z_{1}\right)=13 \mathrm{~cm}$. In fact, the substrate is usually placed at smaller distances of about $10 \mathrm{~cm}$. The presence of the substrate modifies the flow, mainly by diverting the streamlines and forming a thin boundary layer along the surface. The deposition of ultra small particles such as those considered in our paper can happen only via the diffusion in the boundary layer. The other effect of the boundary layer is that the particle velocity strongly decreases, which allows particles to grow larger due to collisions. Nevertheless, the mean size of particles deposited and their composition should be not very different from those obtained in this paper.

\section{Appendix. Physical properties}

Both homogeneous nucleation and heterogeneous condensation are strongly determined by the dependence of the vapour pressure on the temperature. The referenced points for Ta and $\mathrm{C}$ gases used for the simulations are summarized in table A1. One can see that Ta gas starts nucleating at substantially higher temperature than $\mathrm{C}$. Between the data points, the vapour pressure was approximated by the Clausius-Clapeyron equation:

$$
p=p_{\text {ref }} \exp \left[-\frac{Q}{R}\left(\frac{1}{T}-\frac{1}{T_{\text {ref }}}\right)\right] .
$$

Here $p_{\text {ref }}$ and $T_{\text {ref }}$ are the values given in table $\mathrm{A} 1, Q$ is the latent heat of vaporization per mole and $R$ is the universal gas constant. For the latent heat of vaporization the following values were used: for Ta, $Q=7.33 \times 10^{5} \mathrm{~J} \mathrm{~mol}^{-1}$ (Dean 1999) and for C, $Q=3.56 \times 10^{5} \mathrm{~J} \mathrm{~mol}^{-1}$ (Schumm et al 1973).

The size of the critical nucleus is determined by the value of the surface tension. For the homogeneous nucleation of Ta gas, the value of the surface tension coefficient $\sigma=$ $2.07 \mathrm{~N} \mathrm{~m}^{-1}$ reported by Miiller and Cezairliyan (1993) was used. In our calculations, the temperature variations exceed the reported measurement interval. We chose to extrapolate the constant value for the surface tension coefficient on the entire temperature range where the particles exist. Following the common assumption of the nucleation theories we do not consider the dependence of the surface tension on the particle size.

In order to calculate the size of particles, the following diameters of Ta and $\mathrm{C}$ atoms were assumed, $d_{11}=2.7 \times$ $10^{-10} \mathrm{~m}$ and $d_{12}=2.6 \times 10^{-10} \mathrm{~m}$. 


\section{References}

Los Alamos 1964 A Critical Review of Refractories Los Alamos Scientific Laboratory of the University of California, Los Alamos, NM

Basu S and Cetegen B M 2007 Int. J. Heat Mass Transfer 503278

Chen W L T, Heberlein J and Pfender E 1996 Plasma Chem. Plasma Proc. 16635

Dean J A (ed) 1999 Lange's Handbook of Chemistry (15th edn) (New York: McGraw-Hill)

Desilets M, Bilodeau J-F and Proulx P 1997 J. Phys. D: Appl. Phys. 301951

Fetherston J M 2004 Mineral Resources Bulletin 22. Tantalum in Western Australia (Geological Survey of Western Australia)

Fluent 6.3 User's Guide 2006 (Fluent Inc.)

Friedlander S K 2000 Smoke, Dust, and Haze: Fundamentals of Aerosol Dynamics (Oxford: Oxford University Press)

Girshick S L, Chiu C-P and McMurry P H 1990 Aerosol Sci. Technol. 13465

Hafiz J et al 2004 Surf. Coat. Technol. 188-189 364

Ishigaki T, Oh S-M, Li J-G and Park D-W 2005 Sci. Technol. Adv. Mater. 6111

Killinger A, Kuhn M and Gadow R 2006 Surf. Coat. Technol. 2011922

Launder B E and Spalding D B 1972 Lectures in Mathematical Models of Turbulence (New York: Academic)
Launder B E and Spalding D B 1974 Comput. Methods Appl. Mech. Eng. 269

Leider H R, Krikorian O H and Young D A 1973 Carbon 11555

Liu B, Zhang T and Gawne D T 2000 Surf. Coat. Technol. 132202

Mendoza-Gonzalez N Y, Goortani B M and Proulx P 2007 Mater. Sci. Eng. C 271265

McFeaters J S, Stephens R L, Schwerdtfeger P and Liddell M 1994 Plasma Chem. Plasma Proc. 14333

Miiller A P and Cezairliyan A 1993 Int. J. Thermophys. 141063

Murphy A B 2004 J. Phys. D: Appl. Phys. 372841

Nadykto A B and Yu F 2005 J. Chem. Phys. 122104511

Schumm R H, Wagman D D, Bailey S, Evans W H and Parker V B 1973 National Bureau of Standards (USA) Technical Notes $270-1$ to $270-8$

Shigeta M and Watanabe T 2007a Thin Solid Films 5154217

Shigeta M and Watanabe T 2007b J. Phys. D: Appl. Phys. 402407

Shih T-H, Liou W W, Shabbir A, Yang Z and Zhu J 1995 Comput. Fluids 24227

Stull D 1972 American Institute of Physics Handbook 3rd edn ed D E Gray (New York: McGraw-Hill)

Trelles J P, Pfender E and Heberlein J 2006 Plasma Chem. Plasma Proc. 26557

Trignan-Piot L, Berardo M, Gastaldi J and Giorgio S 1996 Surf. Coat. Technol. 79113

Vardelle M, Vardelle A, Fauchais P and Boulos M I 1983 AIChE J. 29236 